\title{
Research Challenges in Environmental Observation and Forecasting Systems
}

\author{
David C. Steere ${ }^{*}$,Antonio Baptista ${ }^{* * *}$,Dylan McNamee ${ }^{*}$, Calton Pu ${ }^{* * * *}$, and Jonathan Walpole \\ *Department of Computer Science and Engineering \\ Oregon Graduate Institute \\ **:Center for Coastal and Land Margin Research \\ Oregon Graduate Institute \\ ****:College of Computing \\ Georgia Institute of Technology
}

\begin{abstract}
We describe Environmental Observation and Forecasting Systems (EOFS), a new class of large-scale distributed system designed to monitor, model, and forecast wide-area physical processes such as river systems. EOFS have strong social relevance in areas such as education, transportation, agriculture, natural resource planning and disaster response. In addition, they represent an opportunity for scientists to study large physical systems to an extent that was not previously possible. Building the next generation of EOFS pose a number of difficult challenges in all aspects of wireless networking, including media protocols for long distance vertical communication through water, flooding algorithms in ad-hoc network topologies, support for rate- and time-sensitive applications, and location-dependent mobile computing.
\end{abstract}

\section{Introduction}

The availability of tremendous computation power coupled with widespread connectivity have fueled the development of real-time environmental observation and forecasting systems (EOFS). These systems couple real-time in-situ monitoring of physical processes with distribution networks that carry data to centralized processing sites. The processing sites run models of the physical processes, possibly in real-time, to predict trends or outcomes using on-line data for model tuning and verification. The forecasts can then be passed back into the physical monitoring network to adapt the monitoring with respect to expected conditions. For example, one could reposition sensors closer to the predicted source of a disturbance to improve sampling accuracy.

EOFS have several unique characteristics that pose interesting challenges in the areas of wireless networking, systems, and mobile computing. In particular, these systems are large-scale, distributed embedded systems in which data primarily flows from remote sensors over wireless links to collection points, and from these to centralized processing via wired links. The system supports a small number of concurrent applications, and like an embedded system can be tuned to meet the needs of the specific workload it is intended to support. The sensor stations can have cost, power, size, and weight constraints, the environment in which they

This project was supported in part by the National Science Foundation grant CCR9876217, DARPA contracts/grants N66001-97C-8522, and N66001-97-C-8523, and by Tektronix, Inc. and Intel Corporation. Early development of CORIE, a reference testbed for this paper, was partially funded by the Office of Naval Research (Grant N00014-96-1-0893) 
run is variable, and the stations may be capable of changing their location. Typically the greater the importance of the sensor, the tighter the constraints under which it must operate. The data flows themselves may be rate- and time-sensitive and as such, steps must be made to ensure quality of service (QoS) for the data.

These characteristics create a number of problems that have not been addressed in the wireless and mobile computing communities, while introducing opportunities for solving these problems. For example, severe power and size constraints on sensor stations coupled with the need for high throughput necessitate the need for adaptive network protocols, yet the fact that these systems are dedicated creates the opportunity to tune protocols to meet the needs of the specific application.

We have built a prototype EOFS, called CORIE, to study the Columbia river estuary and plume, and are now involved in designing its next generation. CORIE[1] consists of sensor stations in the Columbia River Estuary that carry various environmental sensors. These sensors record environmental information, such as temperature, salinity, water levels, and flow velocities, and transmit this information to a centralized compute farm. The sensor information is used to drive 2-D and 3-D fine-grain environmental models. The output of the models has been used for a variety of purposes, including online control of vessels, marine search and rescue, and ecosystem research and management.

The redesign effort has uncovered a number of issues that are not addressed by existing research literature, the goal of this paper is to stimulate research by the wireless networking and mobile computing communities that can ultimately be deployed in future generations of CORIE. The remainder of this paper describes EOFS in more detail, starting with a generic description in Section 2 and then moving to a description of CORIE in Section 3. Section 4 presents a number of potential research topics whose solution would enable a leap in the power and usefulness of EOFS. We strive throughout to avoid biasing the discussion towards particular solutions.

\section{A Description of EOFS}

EOFS are large distributed systems that span wide geographic areas. To simplify the discussion, we assume EOFS have three components: sensor stations, a distribution network, and a centralized processing farm. The stations have one or more sensors, a power supply, and a radio link. The distribution network connects the sensors to the processing farm, possibly using other stations as relays. For purposes of this discussion, the key characteristics of EOFS are:

- Centralized Processing: Sensor stations are cost, power, and weight constrained and so are relatively resource poor. In addition, the scientific applications that utilize EOFS are typically computationally intensive and utilize data from many or all sensors and hence must aggregate the data centrally. In the ideal, EOFS would have large numbers of stations with little or no processing capacity. However, the ability to perform some amount of local processing is very advantageous. For example, one could run models to detect sensor degradation (such as through bio-fouling) before consuming wireless bandwidth to transmit bogus data.

- High data volume: In-situ sensors are capable of generating more data than typical wireless networks can deliver. Sensor stations may have limited processing capacity, reducing their ability to aggressively compress the data. In addition, the highest data rates can correspond to environmental circumstances that are most pessimal for achieving high throughput, such as tsunami. For example, nautical X-band radar used to monitor ocean waves (height, frequency) can generate megabytes of data per second.

- QoS sensitivity: The utility of the data depends on various QoS characteristics, such as end-toend latency and smooth delivery. In addition, control flow may have latency bounds if sensor behavior needs to be coordinated. Limits on bandwidth and processing require degrading some or all of the information flows from the sensors. Ensuring QoS in the face of power constraints and environmental hazards is an open problem.

- Backwards data flow: The primary flow of data originates at the stations and flows back to the servers. In addition, the stations are power constrained, are typically located in remote and 


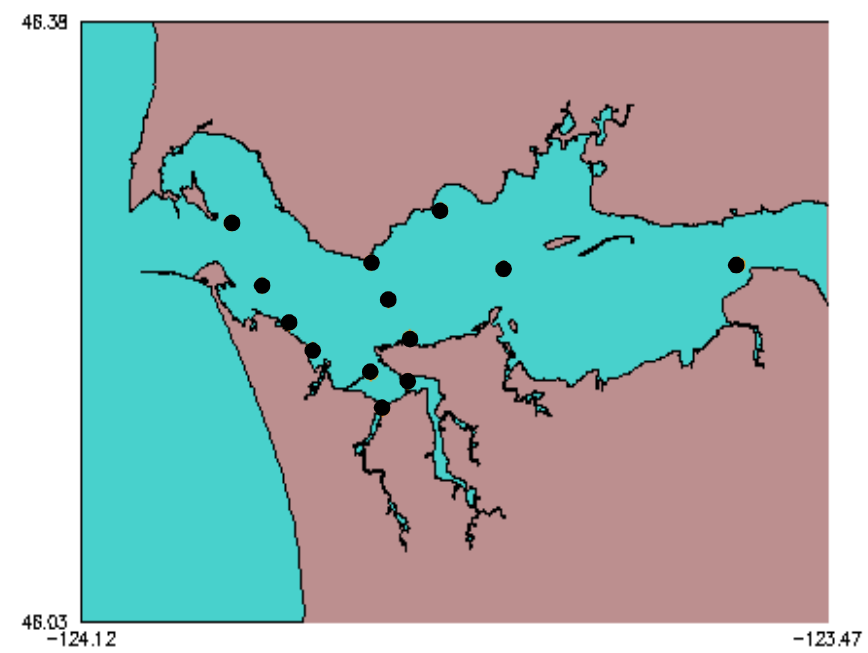

Figure 1: CORIE Station locations in Columbia River Estuary

potentially hazardous locations, and may be mobile. As a result, solutions which require high power transmitters for acceptable signal-tonoise are inappropriate for EOFS. If the stations are mobile, using directional antennas on receivers to pick up weak signals requires automated tracking. This may be complicated if motion is significantly effected by high-frequency perturbations from the environment.

- Extensibility: Given the high cost of deployment, EOFS are most useful when they can serve a variety of scientific applications. This can be achieved by placing a variety of instruments on each station, some subset of which may be in use at any one time. It can be safely assumed that at most a few competing applications will run concurrently.

- Autonomous Operation: The sensor stations are typically placed in remote locations and are difficult or expensive for a human to physically access. As a result they must be extremely robust. The need for reconfiguration implies that the wireless infrastructure must support uploading new applications and code upgrades, as well as the flow of sensor data to the compute servers and the flow of control data back again.

\section{CORIE: an EOFS for the Columbia River}

As an example EOFS, consider the CORIE system built by the Center for Coastal and Land Margin Research at the Oregon Graduate Institute (http://www.ccalmr.ogi.edu). The CORIE monitoring network consists of 13 stations located throughout the Columbia River estuary and one off-shore station located on a buoy. These stations stream data samples to on-shore receivers via a Freewave DGR-115 spread-spectrum wireless network (http://www.freewave.com/dgr115.html), and from there to a centralized compute-farm via a $\mathrm{T} 1$ wired network. CORIE measures various aspects of the Columbia River, including flow field velocity, salinity, temperature and water levels. Figure 1 shows a map of the Columbia river estuary and the location of CORIE sensor stations. Not shown in Figure 1 is the location of the tethered ocean buoy, which is located 10 miles south and 10 miles west of the mouth of the Columbia.

The data from CORIE, plus flow information from upstream dams, is fed to a variety of computation models of coastal circulation (ADCIRC, QUODDY, and POM among others). These models perform 2D and 3D modeling of water circulation and transport. Model output is used both for nowcast, characterizing current conditions over a selected geographic area, and forecast, predicting future conditions such as water depth or flow velocity. 
The basic architecture of CORIE's sensor stations consists of one or more instruments strapped to a fixed object such as a pier or to a tethered buoy. Each instrument can have multiple sensors, such as a conductivity, temperature, and depth gauge, acoustic doppler profiler for measuring flow fields, or nautical x-band radar. Depending on the station, instruments are connected to a field computer via a serial cable. The field computer has a $133 \mathrm{Mhz} 586$ processor, $32 \mathrm{MB}$ of RAM, a hard drive, and a radio modem, all of which are contained in a sealed box. Near-shore stations use power from the electric grid, others rely on solar photovoltaic power. In cloudy conditions, the solar panels are insufficient to charge the battery for continuous operation, so intermittent failure due to power loss is a common occurrence especially in winter.

Each sensor station communicates to a master station via a Freewave 115 Kbaud radio modem, which operates under 1 watt in the 902-928 Mhz band. The master station is near the shore, uses utility electric power, and is accessible for easy servicing. Media access uses a time-division multiple access protocol, manually configured based on instrument number, sampling rate, and location. Some stations do not have direct line-of-sight to the master station, and so their communication must be relayed through other stations. Selection of the master station and the topology of the wireless network can only be changed through manual (and physical) intervention. Currently the failure of a repeating station results in loss of real-time data from the stations using the repeater, although the stations can record some amount of data internally and so the data itself is not typically lost. Reconfiguring the network in response to a station failure is as expensive as replacing the failing unit.

We are currently interested in studying phenomena that require extensions to CORIE. These extensions make our existing networking infrastructure inadequate and motivate this challenges paper.

- Autonomous Mobility: Currently, CORIE's stations are fixed in location and hence we are unable to study mobile or small-scale phenomena and environmental interfaces. Deploying manually controlled vessels to study these phenomena is both expensive and inefficient. We would therefore like to deploy autonomous mobile sensor stations that can follow physical processes, using forecasts from CORIE's mod- els to control sensor location. For example, we are collaborating in a study to investigate the formation, characteristics, and ecological significance of estuarine turbidity maxima (ETM). ETM are an important and very dynamic ecological feature of many estuaries that results from the trapping of large quantities of sediments in the vicinity of a salt wedge. The salt wedge and the ETM form in constrained channels where freshwater from the river meets saltwater from the ocean. ETMs are non-permanent features, with complex but essentially one-directional propagation and with varying intensity over a tidal cycle.In concept, forecasting from systems like CORIE could be used to control a vessel surveying ETM phenomena if the networking issues could be solved.

- Reactive behavior: Events in some regions of the data collection grid or external to the grid may affect other regions. An example is the offshore detection of a tsunami approaching the coastline. If these events are recognized quickly, sensors in the affected regions may be reprogrammed to capture the effects, e.g., water level sensors can be reprogrammed to sample at higher frequencies than normal. Such reprogramming must happen quickly, on the scale of minutes, in order for the reactive behavior to begin before the effects propagate from the event source. In the Pacific Northwest, for instance, Cascadia Subduction Zone tsunamis may take just 5-30 minutes from generation offshore to impact on the coast.

- Time and location dependence: Investigation of environmental issues such as conditions for salmon survival in the ocean may require coordinated sampling, in space and time, by multiple manned and unmanned platforms. We are interested is in having multiple vessels, each performing specific sampling tasks (e.g., lowdensity fish catches or high-density oceanographic measurements), coordinate their sampling strategies among themselves and with information from real-time model forecasts, static buoys, and a large number of passive ocean drifters equipped with temperature and salinity sensors. Ultimately, each "platform" (buoys, drifters, vessels, and models) should 
have the ability to change sampling or computing protocols based on local or remote information. For instance, drifters may control their vertical position in the water column based on information on observed or predicted local density structure. Vessels may try to follow dynamic fronts. Sensors in buoys may change range or density of samples ahead of predicted arrival of the same fronts. Models may change data assimilation procedures based on the changing spatial density of drifters throughout the domain. The integrated management of the mobile observation network is a challenge that we can not address with present technology.

\section{Research Challenges}

We are currently in the process of designing the next-generation of CORIE, and in the process have identified a number of research issues which no satisfactory solution currently exists. The following subsections identify the key problems in this effort. In the interests of space, we have restricted the discussion to research issues involving mobile computing and wireless networking.

\subsection{Adaptability}

A key characteristic of EOFS is that the demand for resources such as computation, battery power, and bandwidth is always higher then the supply. In addition, choosing optimal trade-offs depends on the ultimate use of the sensor data. These two facts indicate the need for adaptability at all levels. In addition, since the EOFS is likely to support one or a few applications at a time, significant benefit can be obtained by allowing low-level (media, link, and transport) mechanisms to be tuned to meet the needs of the application. For example, there is no logistical advantage to using a general purpose transport mechanism like TCP in this environment, except that it is already written and is reasonably bug free.

\subsection{Periodic disruptions in line-of-sight}

After deploying a tethered buoy 15 miles off the Oregon coast, we discovered that the height of surface waves frequently exceeds the height of the antenna on the buoy, obscuring line-of-sight with the receiver on shore. This in turn disrupts commu- nication except when the buoy is riding near the crest of the wave. Unfortunately, existing protocols are not very robust to these disruptions, and as a result we get very little effective bandwidth on the radio link. As a stop-gap solution, we communicate data to an ORBCOMM LEO satellite (http:// www.orbcomm.com/) that propagates it back to our processor farm in the form of email every 30 minutes. This solution severely impacts latency in receiving data, as well as incurring significant cost and power overheads.

A solution to this problem must account for a number of issues. First, the frequency of the surface waves varies, and thus the time between periods of connectivity changes over time. Slight variations in wave height and frequency are difficult to predict accurately given current models. Second, the buoy has very limited power resources. Solutions which require probing or rebroadcasts can severely limit the effective lifetime of the buoy. Third, the buoy has higher bandwidth requirements than the stations closer to shore, since it is sampling data at multiple depths on its tether. Yet it is located further from the base station ( 15 miles), and has a less stable base on which to affix an antenna. Unfortunately, despite these difficulties these sorts of off-shore tethered buoys are likely to be more common and more important in future EOFS. To our knowledge this problem has not been addressed by the research community.

One aspect of mobile computing that would be complicated by this form of periodic outage is adhoc routing (for example [4],[7],[8], or [9]). Consider a fleet of autonomous mobile sensor stations with low profiles travelling through the ocean studying the plume of the Columbia river. The network topology would change frequently as different stations were lifted and lowered by waves, causing constant network reconfiguration. There are two mitigating factors for EOFS. First, the primary flow of information is known: from sensor to shore. Hence the routing algorithm can take physical location (via GPS) into account, and only propagate information to other stations that are closer to shore. Control information that flows from shore to ship could use a similar approach if the nature of the message is known. Second, it may be possible to deploy special flagships with the fleet that are always available for communication. For example, 
the flagships may be tall enough that their antenna height exceeds the average wave height.

\subsection{Efficient Distribution Algorithms}

Distribution of control messages to all sensors/ buoys must occur rapidly for real-time coordinated behavior, while minimizing excess traffic on the wireless channel. Excess traffic has two negative effects. First, it reduces the effective capacity of the wireless link and thus degrades the quality of the incoming data. Second, it increases the power drain on nodes that must repeat the control messages, reducing their effective operating hours. However, some of the applications, including real-time control of mobile vessels to study ETM, end-to-end latency is of critical importance so round-trip propagation delays must be bounded. Small round-trip times overrides the power concerns.

Some work has been done in the area of flooding or distribution algorithms. Heinzelman et al. compare several different distribution algorithms, including flooding, gossiping, and a new algorithm they call SPIN[3]. Each of these algorithms trades time to convergence (all nodes have received the message) for energy dissipation (total energy used to transmit and receive messages). An ideal solution requires knowledge of network topology, in particular a shortest-path spanning tree of the network. There are several ameliorating factors that lead us to believe that we can develop algorithms that will perform better for EOFS. First, in EOFS the network topology is typically known, modulo periodic disruptions discussed in the previous section and intermittent power failures. Second, wireless networks allow for limited broadcast, all stations within receiving range of the transmission can receive the message simultaneously. In addition, it is possible to structure EOFS hierarchically, so that better connected or more stable stations can serve as repeaters for those farther out. Third, some applications may chose to favor time-to-convergence while others favor energy conservation.

\subsection{Low-power, low-cost sensor-to-shore}

One unique characteristic of EOFS is that the primary flow direction is the opposite than traditional distribution networks in that the transmitter and source of the data (the sensor) is the weakest link. One source of the weakness is due to power and cost considerations arising from the need to deploy sensors near the location of the physical phenomena to be studied. Another source of the weakness is the variability of the operating environment in which the stations are placed and the hazards that may be part of that environment. These operating conditions may increase the noise level requiring even higher power consumption to achieve acceptable signal-to-noise for communication.

A solution proposed by Kahn et al. has the sensor station reflect and modulate a signal that originates from the receiver[5]. This requires little power consumption on the part of the sensor itself. However, direct application of their ideas may be impractical in an EOFS such as CORIE. First, the distance between the station and the on-shore receiver may be quite large: the tethered buoy is 15 miles off shore and future buoys may be further away. Second, the sensor stations in an EOFS are unlikely to be as densely arranged as with smart dust, requiring significant accuracy in reflecting the signal back to its origin. Currently, the tethered buoys do drift, are subject to torsional forces that cause them to rotate, and rise and fall with the waves. Hence it may be difficult to achieve sufficient accuracy in the field.

\subsection{High bit-rate acoustic modems}

Recently, scientists have begun to study the relationship between plate tectonics and surface effects. One example is being deployed by the National Oceanic and Atmospheric Administration in the area of the Cascadia Subduction Zone along the Pacific Coast.[6] The purpose of this system is to detect tsunamis and report them in real-time to communities at risk, current mechanisms are plagues by high rates of false alarms. One problem faced by these scientists is communication between ocean-floor sensors and surface stations. The distance between the sensors on the floor and the ocean surface is several kilometers, using cables as communication media is impractical.

Early prototypes of this system used Datasonics ATM-845/851 acoustic modems for communication, which provide 1200 baud on the uplink and 80 baud on the downlink. Another experiment with the same modem pair found that in typical conditions, maximal throughput on the uplink was lim- 
ited to $300 \mathrm{bps}$ to $600 \mathrm{bps}$ with error loss less than $25 \%$, and downlink bandwidth was limited to 40 bps.[10] Although these link capacities are acceptable for command messages or occasional notification, they are not sufficient to support significant data collection.

\section{Existing Work}

CORIE is just one example of an EOFS that could benefit from addressing the research challenges outlined in this paper. Another example EOFS (referred to above) is being deployed by the Pacific Marine Environmental Laboratory of the National Oceanic and Atmospheric Administration to detect tsunami and warm coastal communities of the impending danger.[6] This EOFS consists of ocean floor bottom pressure recorders (BPRs) that detect sudden changes in water pressure and relay pressure readings to a moored buoy on the ocean surface via an acoustic modem. The buoy then relays the signal to the shore via satellite. As mentioned in Section 4.5, early experience with their prototype indicate the need for better technologies for communicating between ocean floor and surface.

Another class of EOFS maintained by NOAA, with sites in S. Francisco Bay, Tampa Bay, Chesapeake Bay and other coastal waterways, is the Physical Oceanography Real-Time System (PORTS, http://co-ops.nos.noaa.gov/d_ports.html). PORTS supports safe and cost-efficient navigation by providing ship masters and pilots with accurate real-time information required to avoid groundings and collisions, and may ultimately be the basis of for a vessel traffic system for waterways similar in concept to that used in aviation.

EOFS are similar in nature to sensor networks previously described in MOBICOM challenge papers. Estrin et al. describe sensor networks in which sensor identity or address is not needed by the consumer of the data a sensor generates, and discuss the need for decentralized, or "localized" algorithms which can operate without centralized control. By contrast, the chief processing in EOFS, computing flow dynamics in real-time, must be centralized as it involves inputs from many sensors and the computation required greatly exceeds the processing capacity available at the sensors. In addition, the location/address and identity of a sensor matters to the computation.

Kahn et al. describe a network of MEMS-based sensors[5]. These sensors are microscopic, selfcontained, and have limited lifespans. Networks of these sensors are massive in scale, and sensor density is likely to be quite large. By comparison, EOFS sensor stations are large and sparsely distributed. As a result, networking technologies for smart dust are unlikely to be appropriate for EOFS. One area of overlap is the use of remotely controlled mobile vehicles, such as drogues. We have run experiments letting drogues be carried by marine currents, in much the same way that Kahn's smart dust is carried by air currents. However, drogues are sufficiently large that more traditional networking technologies are likely to apply.

\section{Conclusions}

In this paper we have described a new class of distributed sensor networks called Environmental Observation and Forecast Systems. These systems collect streams of instrument data from in-situ sensor stations over multi-hop wireless networks, and feed this data to computationally intensive physical models to produce nowcast/forecasts of the physical processes. We discussed characteristics of EOFS that differentiate them from traditional distributed systems, and from descriptions of other sensor networks, and presented an example EOFS that has been built to study the Columbia River Estuary. We also presented several specific research problems whose solution will enable the next generation of EOFS.

\section{Acknowledgments}

CORIE is maintained, under the supervision of the second author, by a team that includes Michael Wilkin, Cole McCandlish, Dr. Edward Myers, Dr. Arun Chawla, Philip Pearson, Marc Drage and John Graves. Early development of CORIE (Jun 96-Sep 98) was partially funded by the Office of Naval Research (Grant N00014-96-1-0893). Applications of CORIE have been partially funded by the National Science Foundation (LMER, EGB and SGER programs), Bonneville Power Administration, National Marine Fisheries Service, Defense Advanced Research Projects Agency (Software Enabled Control Program) and Office of Naval 
Research (Modeling and Prediction Program). The development and maintenance of a system like CORIE requires strong community support. Thanks are due the Clatsop Community College, U.S. Coast Guard, Northwest River Forecast Center, U.S. Geological Survey, Oregon Department of Transportation, Coastal Studies and Technology Center, U.S. Army Corps of Engineers, Port of Portland, City of Astoria, Columbia Pacific Community Information Center, and Capt. R. Johnson (Columbia River Bar Pilots). We would also like to thank the anonymous reviewers for their helpful and insightful comments.

\section{References}

[1] A. M. Baptista, M. Wilkin, P. Pearson, P. Turner, C. McCandlish, and P. Barrett. Coastal and Estuarine Forecast Systems: A Multi-Purpose Infrastructure for the Columbia River. Earth System Monitor, 9(3), National Oceanic and Atmospheric Administration, Washington, D. C., March 1999.

[2] D. Estrin, R. Govindan, J. Heidemann, S. Kumar. Next Century Challenges: Scalable Coordination in Sensor Networks. In Proceedings of the Fifth Annual ACM/IEEE International Conference on Mobile Computing and Networking. Seattle, August 1999.

[3]W. R. Heinzelman, J. Kulik, and H. Balakrishnan. Adaptive Protocols for Information Dissemination in Wireless Sensor Networks. In Proceedings of the Fifth Annual ACM/IEEE International Conference on Mobile Computing and Networking. Seattle, August 1999.

[4]D. B. Johnson and D. A. Maltz. Dynamic Source Routing in Ad Hoc Wireless Networks. In Mobile Computing. Tomasz Imielinski and Henry F. Korth, editors. Kluwer Academic Publishing, 1996.

[5] J. M. Kahn, R. H. Katz, and K. S. J. Pister. Next
Century Challenges: Mobile Networking for "Smart Dust". In Proceedings of the Fifth Annual ACM/IEEE International Conference on Mobile Computing and Networking. Seattle, August 1999.

[6] H. B. Milburn, A. I. Makamua, F. I. Gonzalez. RealTime Tsunami Reporting from the Deep Ocean. 1996. http://www.pmel.noaa.gov/tsunami/ milburn1996.html

[7]S. Murthy and J.J. Garcia-Luna-Aceves. An Efficient Routing Protocol for Wireless Networks. ACM Mobile Networks and Applications Journal, Special Issue on Routing in Mobile Communications Networks, 1996.

[8]C. E. Perkins and P. Bhagwat. Routing over multi-hop wireless networks of mobile computers. In Mobile Computing. Tomasz Imielinski and Henry F. Korth, editors. Kluwer Academic Publishing, 1996.

[9]S. Singh, M. Woo, and C. S. Raghavendra. PowerAware Routing in Mobile Ad Hoc Networks. In Proceedings of the Fourth Annual ACM/IEEE International Conference on Mobile Computing and Networking. Dallas, October 1998.

[10]R.A. Stephen, G.L. Austin, S.T. Bolmer, C.D. Chadwell, J.A. Collins, D.M. Jabson, P. Jonke, R.G. Goldsborough, M.R. Gould, J.A. Hildebrand, C.B. Hollinshead, D.G. Offield, J.A. Orcutt, K.R. Peal, D.V. Price, S.G. Rosenblad, F.N. Spiess, F.L. Vernon, D.F. Willoughby, and F.B. Wooding. A Pilot Experiment for Broadband Seismic Measurements on the Deep Ocean Floor, The Ocean Seismic Network Pilot Experiment Deployment Cruise, Report for Cruise TN074 on the R/V Thomas G. Thompson. January 3 February 11, 1998, Honolulu - Honolulu. http:// autumn.whoi.edu/osn/0aa_Front_page.html. See Section 3.g, Performance Review of the Acoustic Modem Communications Link during OSN1 BBOBS Instrument Deployments. http:// autumn.whoi.edu/osn/3g_Modem_GO.html 